\title{
COEO Phase locking and performance optimisation
}

\author{
R. Khayatzadeh, V. Auroux, A. Fernandez, O. Llopis \\ LAAS-CNRS, Université de Toulouse, CNRS, UPS, Toulouse, France \\ 7 avenue du Colonel Roche, 31031, Toulouse, France \\ rkhayatz@laas.fr
}

\begin{abstract}
In this paper, a coupled optoelectronic oscillator (COEO) is phase locked to a low noise RF oscillator in order to reduce the phase noise close to the carrier. Biasing current of the optical semiconductor amplifier (SOA) and a varactor diode in the RF part of the COEO are used as two different actuators to perform phase locking. The lock on the optical device is finally simpler and more efficient than the lock on the RF loop. In the second part of this paper, phase noise of the COEO is reduced by more than $15 \mathrm{~dB}$ using a chirped Bragg filter that compensates the dispersion of the optical loop.
\end{abstract}

Keywords- Microwave oscillator; microwave optics; optoelectronic oscillator; phase noise; phase locking

\section{INTRODUCTION}

The significant advance in telecommunication and signal processing systems in the past decades has increased the requirement for high spectral purity microwave and millimeter wave oscillators. Frequency multiplication from highly stable sources, such as quartz sources, is limited by the increase of the noise floor, which is often prohibitive at millimeter wave frequencies. On the other side, convergence of microwave and photonics techniques become very popular to generate highly stable signals in this frequency range [1-3]. Coupled optoelectronic oscillator (COEO) is one of the well-known microwave-photonics techniques able to produce spectrally pure radio frequency and millimeter-wave signals [4], [5]. The main idea behind the COEO is based on the optoelectronic oscillator (OEO) which uses optical storage energy element to achive high spectral purity [3] and [6]. However, in the COEO approach, the laser source and the resonator become the same device. The advantages are the following: no locking circuit is required and a very high equivalent $\mathrm{Q}$ factor and efficient spurious mode suppresion can be achieved [4], [5] and [7-9]. The COEO behaves as a resonator based OEO, because the optical signal is going through an optical loop (which increases the effective $\mathrm{Q}$ factor) but with an optical source which is part of the system and which intimately depends on the RF signal it generates.

If the devices included in the COEO loops are well chosen, and particularly the active devices (optical and microwave amplifiers), and if the fiber length and dispersion parameters are optimized, low phase noise operation can be achieved. The design of our $10 \mathrm{GHz}$ OEO has already been described elsewhere [10], and thus only the key elements are given here. The phase noise obtained with this set-up is good, but the oscillator remains sensitive at very low offset frequencies to vibrations or thermal fluctuations. Therefore, it is interesting to investigate on the stabilization of the COEO frequency using small bandwidth loops and a quartz-referenced source.

In this work, the $10 \mathrm{GHz}$ COEO is phase locked to a low noise local oscillator in order to reduce the close-in phase noise value. In this approach, two different actuators, one in the RF part and one in the optical part, are tested. The first actuator is the bias current of the semiconductor optical amplifier (SOA) included in the mode-locked laser. The bias current is modulated by the command signal from the servo loop. Recently, there have been some publications on RF phase shifters based on current control of an SOA in a microwave photonic system [11], [14]. This approach relies on Coherent Population Oscillations (CPO) in semiconductor structures [12], [13]. The basic working principle of this approach is to change the group velocity using a sharp variation of the refractive index. This variation of the refractive index originates from the population oscillation induced by the pump and probe signals in a two level system. Based on this approach, biasing current of an SOA allows one to control the delay and phase shift of the RF output signal.

The second actuator is a varactor diode that is placed into the RF part of the COEO. This voltage controlled capacitance is connected in series and changes the phase of the RF signal before the electro optical modulator.

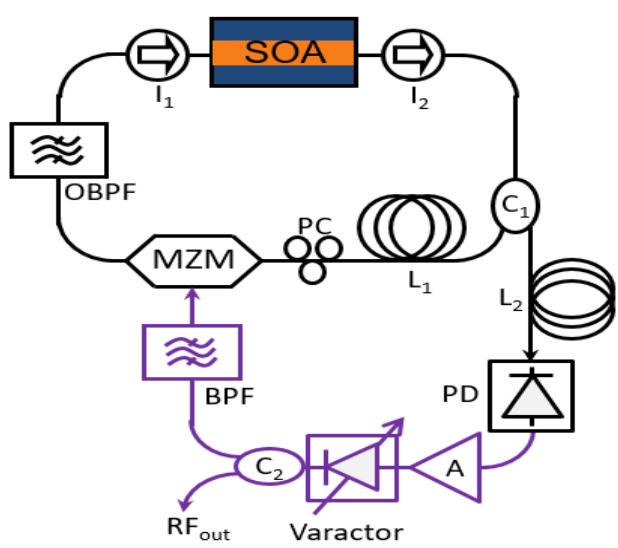

Fig. 1. Experimental setup. SOA: Semiconductor optical amplifier, MZM: Mach-Zehnder modulator, PD: Photodiode, PC: Polarization controller, OBPF: Optical Band-pass filter, BPF: Band-pass filter , C1: optical coupler, C2: RF coupler.

Using this phase locking approach, the phase noise value at $10 \mathrm{~Hz}$ offset frequency is reduced by a factor of more than $20 \mathrm{~dB}$ and $30 \mathrm{~dB}$ for the RF actuator (varactor) and optical 
actuator (biasing current of SOA), respectively. In the first step, a simulation on MATLAB Simulink is presented in order to model the phase locking conditions of the COEO from a system point of view.

In the second part of this paper, a dispersion compensation technique using a chirped Bragg filter is presented. Using this technique, the phase noise of the COEO is reduced by $10 \mathrm{~dB}$ at $10 \mathrm{kHz}$ offset frequency. The advantage of using a Bragg filter is that the group velocity dispersion of the optical fiber can be compensated independently without changing the optical cavity length. Whereas in the techniques based on dispersion compensating fibers (DCF), one needs to add some meters of DCF fiber in the optical loop. Therefore, the free spectral range (FSR) of the optical cavity will be changed.

\section{SIMULATION OF LONG TERM STABILIZATION}

The schematic diagram of the system is presented in Fig. 2. The coupled optoelectronic oscillator (COEO) system is considered as a first order system with the response time of one millisecond. The gain of this system is measured and is equal to $50 \mathrm{~Hz} / \mathrm{mA}$ (current actuator). The output frequency of the COEO is set to an arbitrary value (for example $9.9 \mathrm{GHz}$ ) and the aim is to reach to the frequency of the Quartz (for example $10 \mathrm{GHz}$ ). An integrator is placed just after the COEO frequency in order to transform frequency to phase. An integrator is also used for the Quartz to transform the output frequency to phase. We need to mention that these integrators are not needed in real experimental realization. However, since in real experiments we use phase detector to phase lock the two oscillators we need to transform frequency to phase. The phase detector constant is $200 \mathrm{mV} / \mathrm{rad}$.

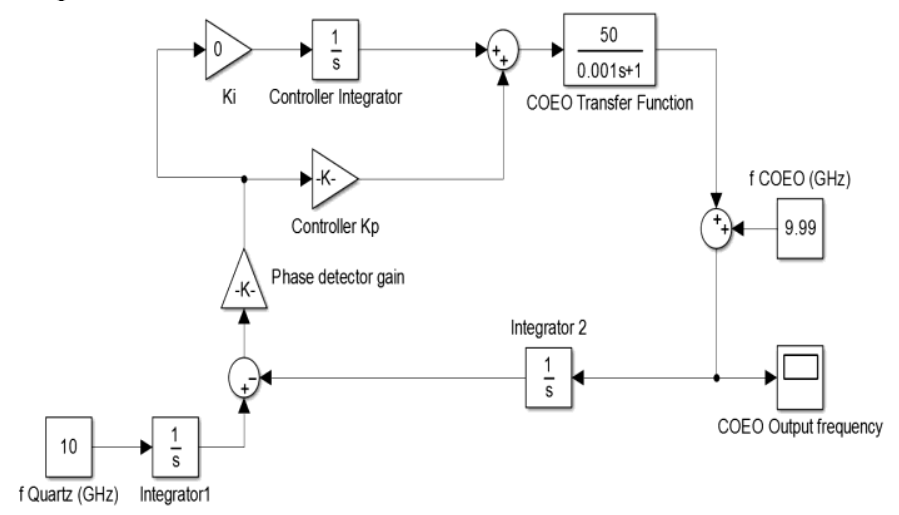

Fig. 2. Schematic diagram of COEO phase locking simulation MATLAB Simulink.

The simulation results for different proportional gain values (Kp) are presented in Fig. 2. In this simulation the integrator gain is set to zero. As can be seen in this figure, the COEO oscillator frequency reaches $10 \mathrm{GHz}$, which means that it is smoothly locked to the quartz.

\section{EXPERIMENTAL SETUP OF LONG TERM STABILIZATION}

Fig.1 presents the architecture of the COEO. In this figure, the optical parts and the RF parts are shown by black lines and violet lines, respectively. The SOA is chosen because of its

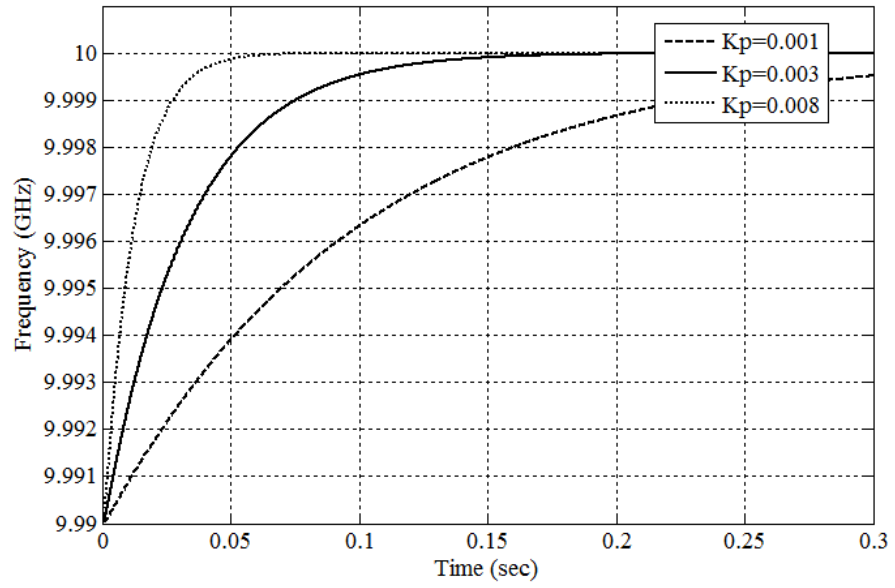

Fig. 3. COEO phase locking simulation MATLAB Simulink results.

compact size and low close to carrier phase noise. The SOA features an output saturation power of $16 \mathrm{dBm}$ and a $30 \mathrm{~dB}$ small signal gain. In order to reduce the spontaneous emission of the SOA a $2 \mathrm{~nm}$ optical filter is placed in the optical loop. The optical delay line (L1) is a dispersion compensating fiber (DCF) of $100 \mathrm{~m}$ combined with $400 \mathrm{~m}$ standard single mode fiber (SMF). The optical delay line (L2) is a single mode fiber of $150 \mathrm{~m}$. The second fiber delay (L2) before the fast photodiode enables both the filtering of side modes through Vernier effect and the increase of the optoelectronic loop quality factor. A polarization controller is placed before the modulator to control the polarization of the light at the input of the $40 \mathrm{GHz}$ bandwidth MZM. The RF beat note is amplified, filtered and sent back to the MZM using the RF feedback part. The filter used in this work is a dielectric resonator centered at $10 \mathrm{GHz}$ and features a loaded quality factor of 2500. The varactor placed in the RF part can be used either to adjust the $\mathrm{RF}$ phase with a DC voltage (compensation of a drift) or to phase lock the COEO.

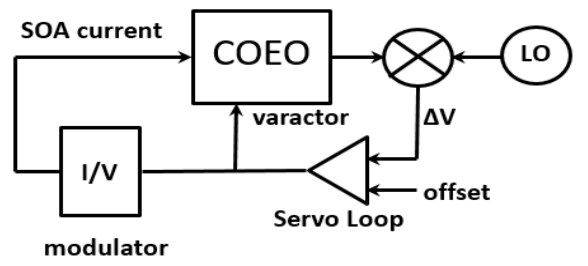

Fig. 4. Schematic diagram of the phase locking system. LO: Local oscillator.

Fig. 4 shows the schematic diagram of the COEO phase locking system. The local oscillator (LO) is a low phase noise Anritsu signal generator. The error signal $(\Delta \mathrm{V})$ at the output of the mixer (phase detector) is added to an offset signal and sent to a variable gain servo loop $(0<\mathrm{G}<40 \mathrm{~dB})$ and the control signal at the output of the servo loop is sent to one of the two actuators. In case of using current actuator, the command signal is sent to the current modulator of the current controller module (ILX LDC3900). The COEO tuning coefficient obtained with the current is measured and is equal to $50 \mathrm{~Hz} / \mathrm{mA}$. The tuning coefficient obtained with the varactor 
diode is not linear. It is of $450 \mathrm{~Hz} / \mathrm{V}$ near $0 \mathrm{~V}$, and drops at $150 \mathrm{~Hz} / \mathrm{V}$ near $4 \mathrm{~V}$ offset.

\section{RESULTS AND DISCUSSION}

Fig. 5 depicts the measured phase noise for three different scenarios. The black curve presents the phase noise of the free running COEO. The grey curve shows the measured phase noise of the COEO locked on low noise LO using current modulation of the SOA and the brown curve shows the phase noise results for the COEO locked on LO using varactor. As it is shown in this figure, the phase noise value for the locking scenarios at close-in offset frequencies are hugely reduced. This phase noise reduction at $10 \mathrm{~Hz}$ offset frequency for the scenario in which the current is used as the actuator is more than $30 \mathrm{~dB}$ and for the scenario in which the varactor is used is more than $20 \mathrm{~dB}$. The locking bandwidth for the current actuator is around $500 \mathrm{~Hz}$ and around $100 \mathrm{~Hz}$ for the varactor approach. In any case, the locking bandwidth is limited by the $\mathrm{Q}$ factor of the COEO. The measured microwave $\mathrm{Q}$ of this system is in the range of $210^{6}$, which corresponds to a halfbandwidth of $2.6 \mathrm{kHz}$.

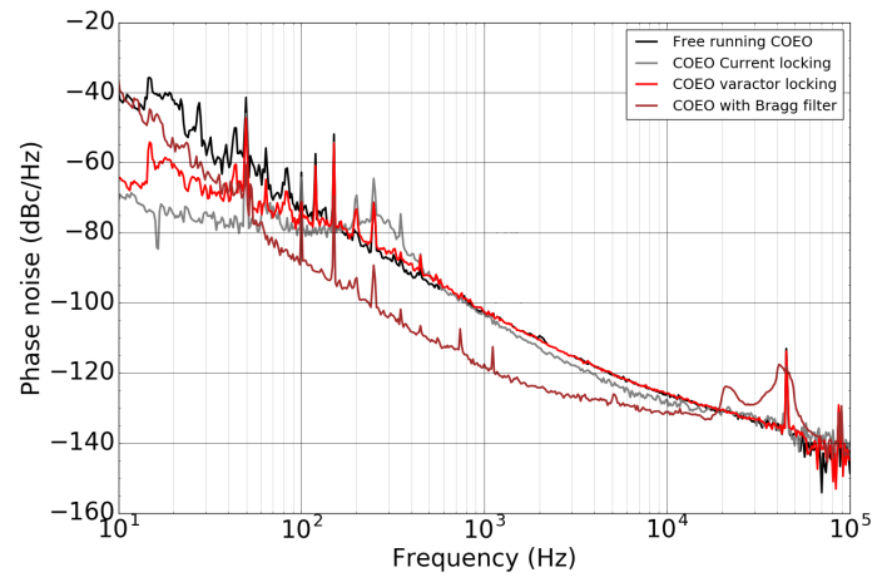

Fig. 5. Phase noise measurement results: free running COEO (black curve), COEO phase locked on LO using current modulation of SOA (gray curve), COEO phase locked on LO using varactor (red curve) and COEO using Bragg filter (brown curve).

\section{DISPERSION OPTIMIZATION}

Fig. 6 presents the schematic diagram of the experimental COEO based on chirped Bragg filter. As can be seen in this figure, the OBPF and DCF are replaced by a chirped fiber Bragg grating (CFBG) filter with chromatic dispersion of -7 $\mathrm{ps} / \mathrm{nm}$ and . Considering $400 \mathrm{~m} \mathrm{SMF}$ fiber with chromatic dispersion of $17 \mathrm{ps} /(\mathrm{nm} . \mathrm{km})$ in the optical cavity, we can compensate the GVD utilizing the mentioned CFBG. Another advantage of using a CFBG is that one can remove the spontaneous emission of the SOA which is a large band amplifier.

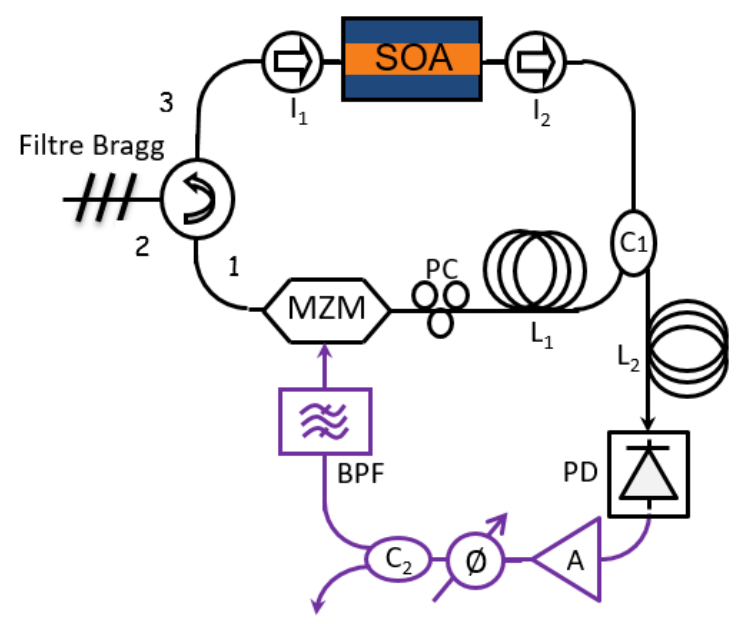

Fig. 6 Experimental setup of the COEO with a chirped Bragg filter.

The phase noise measurement result for the COEO based on Bragg filter is presented in Fig. 5 (brown curve). As can be seen in this figure, the phase noise PSD close to the carrier is hugely reduced. For example at $1 \mathrm{kHz}$ offset frequency the phase noise value of $-120 \mathrm{dBc} / \mathrm{Hz}$ is obtained which is $15 \mathrm{~dB}$ better than the previous results.

\section{CONCLUSION}

In this work, a $10 \mathrm{GHz}$ COEO is phase locked to a low noise RF oscillator using two different actuators from optical and RF parts of the COEO. The optical actuator is the bias current of the SOA and the RF actuator is a varactor that is placed in the RF feedback part of the COEO. The optical actuator approach appears to be the more efficient one. It is also readily available on any system of this type, and thus very easy to set-up. A dispersion compensation technique utilizing a chirped Bragg filter is also presented in order to optimize the performance of the COEO. Using this technique, a phase noise reduction of more than $15 \mathrm{~dB}$ at $1 \mathrm{kHz}$ off-set frequency is observed.

\section{REFERENCES}

[1] J. Yao, «Microwave Photonics,» J. Lightw. Technol., vol. 27, n. 13, pp. 314-335, 2009.

[2] L. Maleki, «The optoelectronic oscillator,» Nature Photonics, vol. 5, pp. 728-730, 2011.

[3] S. Yao and L. Maleki, «Optoelectronic microwave oscillator,» J. Opt. Soc. B, vol. 13, pp. 1725-1735, 1996.

[4] S. Yao and L. Maleki, «Dual microwave and optical oscillator,» Opt. Lett., vol. 22, pp. 1867-1869, 1997.

[5] E. Salik, N. Yu and L. Maleki, «An ultralow phase noise coupled optoelectronic oscillator,» IEEE Photon. Technol. Lett., vol. 19, n. 16, pp. 444-446, 2007.

[6] K. Saleh, P. H. Merrer, O. Llopis and G. Cibiel, «Optoelectronic Oscillator Based on fiber Ring Resonator: Overall System Optimization and Phase Noise Reduction,» in IEEE-Int. Frequency Control Symposium, Baltimore, 2012.

[7] N. Yu, E. Salik and L. Maleki, «Ultralow-noise mode-locked laser with coupled optoelectronic oscillator configuration,» Opt. Lett., vol. 30, n. 110, pp. 1231-1233, 2005.
This work is supported in part by the Direction Générale de l'Arrmement (French MoD) and by Région Occitanie. 
[8] F. Quinlan, C. Williams, S. Ozharar, S. Gee and P. Delfyett, «Selfstabilization of the optical frequencies and the pulserepetition rate in a coupled optoelectronic oscillator,» J. Lightw. Technol., vol. 26, n. 115, pp. 2571-2577, 2008.

[9] S. Yao, L. Davis, and L. Maleki, «Coupled optoelectronic oscillator for generating both RF signal and optical pulses,» J. Lightw. Technol., vol. 18, n. 1, pp. 73-78, 2000.

[10] V. Auroux, A. Fernandez, O. Llopis, P. Beaure d'Augères and A. Vouzellaud, «Coupled optoelectronic oscillators: design and performance comparison at $10 \mathrm{GHz}$ and $30 \mathrm{GHz}$ » in IEEE-Int. Frequency Control Symposium, New Orleans, 2016.

[11] P. Berger, M. Alouini, J. Bourderionnet, F. Bretenaker and D. Dolfi « Slow light using semiconductor optical amplifiers: model and noise characteristics,» C. R. Physique, vol. 10, pp. 991-999, 2009.

[12] P.-C. Ku, et al., «Slow light in semiconductor quantum wells,» Opt. Lett. , vol. 29, pp. 2291-2293, 2004.

[13] J. Mørk, et al., «Slow light in a semiconductor waveguide at gigahertz frequencies,» Opt. Express, vol. 13, pp. 8136-8145, 2005.

[14] P. Berger et al., «intermodulation distortion in microwave phase shifters based on slow and fast light propagation in semiconductor optical amplifiers,» Opt. Lett., vol. 35, pp. 2762-2764, 2010. 